
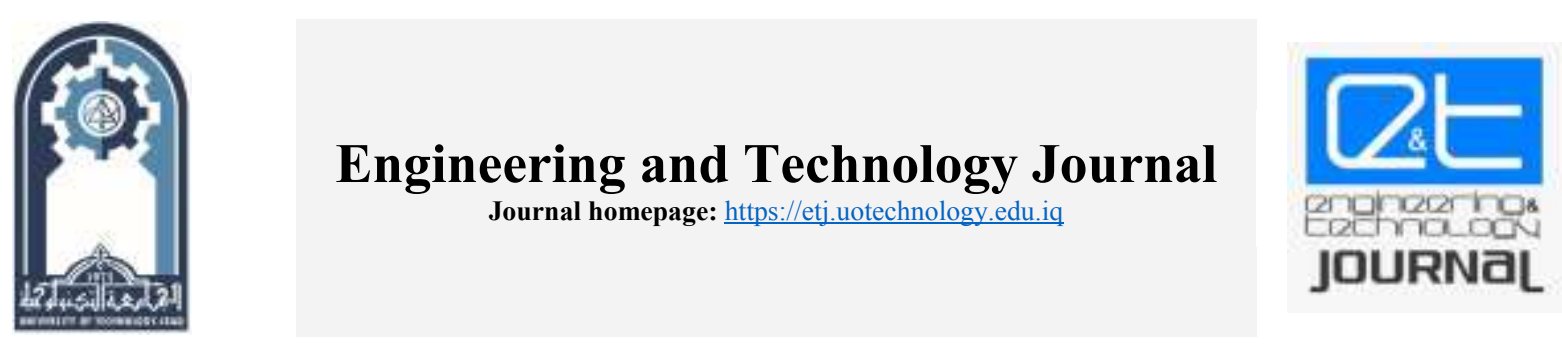

\title{
Water Flow Simulation of Tigris River Between Samara and Baghdad Based on HEC-RAS Model
}

\author{
Zahraa Majeed, Ali S. Abbas, Imzahim A. Alwan* \\ Civil Engineering Dept., University of Technology-Iraq, Alsina'a street, 10066 Baghdad, Iraq. \\ *Corresponding author Email: Imzahim.a.alwan@,uotechnology.edu.iq
}

\section{H I G H L I G H T S}

- Model has been operated to steady state based on the recorded discharge.

- Model has been operated to unsteady state based on the recorded discharge (2012-2017) years.

- HEC-RAS can use successfully for modelling the ecosystem behaviour of Tigris River within a steady area.

\section{A R T I C L E I N F O}

Handling editor: Wasan I. Khalil

Keywords:

Tigris River

DEM

HEC-RAS

Simulation Model

\section{A B S T R A C T}

This work aims to use the digital elevation model (DEM) or 3-Dimensional surface to model and analyze the water flow in the Tigris River. The first stage of this study is based on filed data acquisition survey at 38 stations. These stations were adopted to implement the one-dimensional steady and unsteady numerical flow models. The HEC-RAS software was used to implement these models. The most important results of the steady-state model were that the maximum discharge and velocity and minimum flow area were $638.17 \mathrm{~m}^{3} / \mathrm{sec}, 2.12 \mathrm{~m} / \mathrm{sec}$ and $301.42 \mathrm{~m}^{2}$ respectively. These values occurred at station $66+700$. While, the minimum discharge and velocity and maximum flow area were $504.5 \mathrm{~m}^{3} / \mathrm{sec}$, $0.13 \mathrm{~m} / \mathrm{sec}$ and $4758.13 \mathrm{~m}^{2}$ respectively. The results of unsteady state were analyzed based on the recorded discharge in the year 2018. The maximum and minimum discharge (flow velocity) were $638.12 \mathrm{~m}^{3} / \mathrm{sec}(2.42 \mathrm{~m} / \mathrm{sec})$ at the stations $94+666+7$ and $635.57 \mathrm{~m}^{3} / \mathrm{sec}(0.14 \mathrm{~m} / \mathrm{sec})$ at station $00+00$, respectively. The maximum and minimum Froude number were 0.52 and 0.02 at stations $94+666+7$ and $04+800$, respectively. These results help to identify the flow choking areas that cause defects. In addition, it can be the base for determining the critical sedimentation stations in which the bed level rises and aggravate the flow choking problem within the considered reach of the Tigris River.

\section{Introduction}

Water is one of the primary natural sources for consumption, fish breeding, entertainment, etc. Therefore, traditionally, humans have always been utilizing water resources [1]. Surface water, especially across large cities, is exposed to discharge of numerous contamination sources. These contaminants can alter the balance of the ecosystems [2]. Due to rapid development and urbanization, water scarcity and pollution have significantly affected the availability and quality of water resources. The simulation model of river are the latest techniques by which the behavior of rivers and water characteristics can be studies in a precise and clear manner. These models can provide us the characteristics of water within the river such as velocity, flow, and level. It also gives accurate result for the geometric characteristics of the shape of river, such as longitudinal profile and cross sections, their specifications and areas of strength and weakness. Recently, the use of simulation models, such as HEC-RAS, DEM and 3-Dimensional surface programs have become valuable alternative in most studies. Al-Khafaji [3] evaluated the hydraulic performance of Almsharah River, in the South of Iraq, via using the HEC-RAS software with aid of filed measurements. Nama [4] used HEC-RAS to estimate the sediment transport capacity of Tigris River within the Mosul City. Furthermore, Nama and Abdulhusain [5] utilized HEC-RAS to estimate the riverbed scour due to accumulation of floating debris on Almsharah Bridge piers. Also, Nama [6] determined the distribution of shear stress in the meandering of Tigris River within Baghdad City via using the HEC-RAS and GIS. (Abdelbasset et al. ) [7] built hydraulic style by using program HECRAS and GIS, where they managed to calculate water flow currents through the Al-Wahada Dam. The result showed that the HEC-RAS and GIS model can be used successfully in Simulation results based on constructed model profile and cross section of water elevation and moping of flooded area. (Dang and Kumar ) [8] studied the techniques of remote sensing using GIS to generate a hydrological model to calculate the hazard of flooding on the marshy of the Meakong Delta in Chai Minha City. The result showed that the GIS model can be used successfully in simulation based on constructed model to generate a hydrological model to calculate the hazard of flooding in the urban area. Sharma and Mujumdar [9] used one-dimensional 
model HEC-RAS and two-dimensional model CCHE2D to simulate the flood zoning in the Sungai Maka district in Kelantan state, Malaysia. The results of these two models in most sections approximately Similar but the differences were in the shape of the river. Traore et al. [10] used GIS to build DEM for study river basin in Kayanga, Senegal. It is located between the Kounkane threshold and Niandouba Dam. The result showed that the GIS model can be used successfully in Simulation results based on constructed model. Onea and Bagatur [11] have predicted flood frequency factor for Gumbel distribution with regression and GEP models. Bagatu and Onen [12] have presented the development of a predictive model for flood routing using genetic expression programming. Romali et al. [13] presented application of HEC-RAS and ARC GIS for floodplain mapping in Segamat town, Malaysia. Khalfallah and Saidi [14] have presented spatiotemporal floodplain mapping and prediction using HEC-RAS-GIS tools in the Mejerda River, Tunisia. The main objective of this study is to demonstrate the ability of simulation model to recognize behavior of rivers and water characteristics can be studied in a precise and clear manner. Alwan, Samueel, and Abdullah , [14] studied the accuracy of different elevation model; The area of study is located East of the Amarah city (Maysan governorate) in the South of Iraq which is outlying the capital (Baghdad), The results showed that the ALOS V1.1 model is the most accurate of the open-source models followed by the SRTM V3 model and then followed by ASTER V2. The results obtained from a pair by Pleiades high resolution (PHR) 1B satellites show a higher accuracy than the results obtained from the open-source models.

Maatooq and Hameed ,[15]studied a series of experiments runs were carried out through combining different geometric and hydraulic parameters to produce different experiment conditions. These parameters are flow rate, bed slope, and different initial incised and wide channels for both rectangular and trapezoidal sections. Karim and Sahib ,[16]in this study the EPANET software was used for automatically solving problems of the network. The main objective of this study is to analyze the irrigation network of the proposed Taq-Taq Dam using hydraulic simulation software, the results concluded that the simulated model seems to be reasonably close to those of an actual network system. The study area was selected based on its importance in terms of the amount of inflow water, representing a good example of the ecological system. Simulation models need to define the characteristics of the study areas in a precise way to provide way to need to define the characteristics of the study area in a precise way to provide accurate results, in particular the definition of the geometric characteristics of the rivers of study area, which were represented in this study by the main rivers branch of the Tigris river in the Salah Al-Din Governorate. Using HEC-RAS mathematic model is a method to determining water level and hydraulic specifications of flow. Therefore, the parameter of water surface profile, within time of maximum discharge, for steady and unsteady flow condition, have calculated. This calculation, determine the hydraulic specification of flow, such as, flow depth, critical depth, flow energy and other hydraulic parameters of zone [17]. In this paper, four corrected satellite images for the year 2015 were used to produce the path of these river and their banks from both sides.

\section{Materials And Methods}

\subsection{Study Area and data collection}

The Tigris River is one of the largest rivers in the Middle East, stretching for over $1900 \mathrm{~km}$, of which $1415 \mathrm{~km}$ are within Iraq, the catchment area reaches to $235,000 \mathrm{~km}^{2}$. Tigris River sharing with Euphrates River are the main source for man's use, especially for drinking water since they pass the major cities in the country [16]. In this paper, it begins the study area of the Tigris River from Samarra barrage-to Baghdad city-Resafa station show as the Figure (1). The data collection in this study data are important and spatial of two categories: spatial data and attribute data. Spatial data illustrate the river location with geographical characteristics, whereas attribute data describe and represent spatial data as number or phrases. The plan data, geometric, flow data, and hydraulic design data are used for modeling with HEC-RAS model. The basic input data requirements to run one-dimension include river location coordinates, river cross sections, and initial water surface.

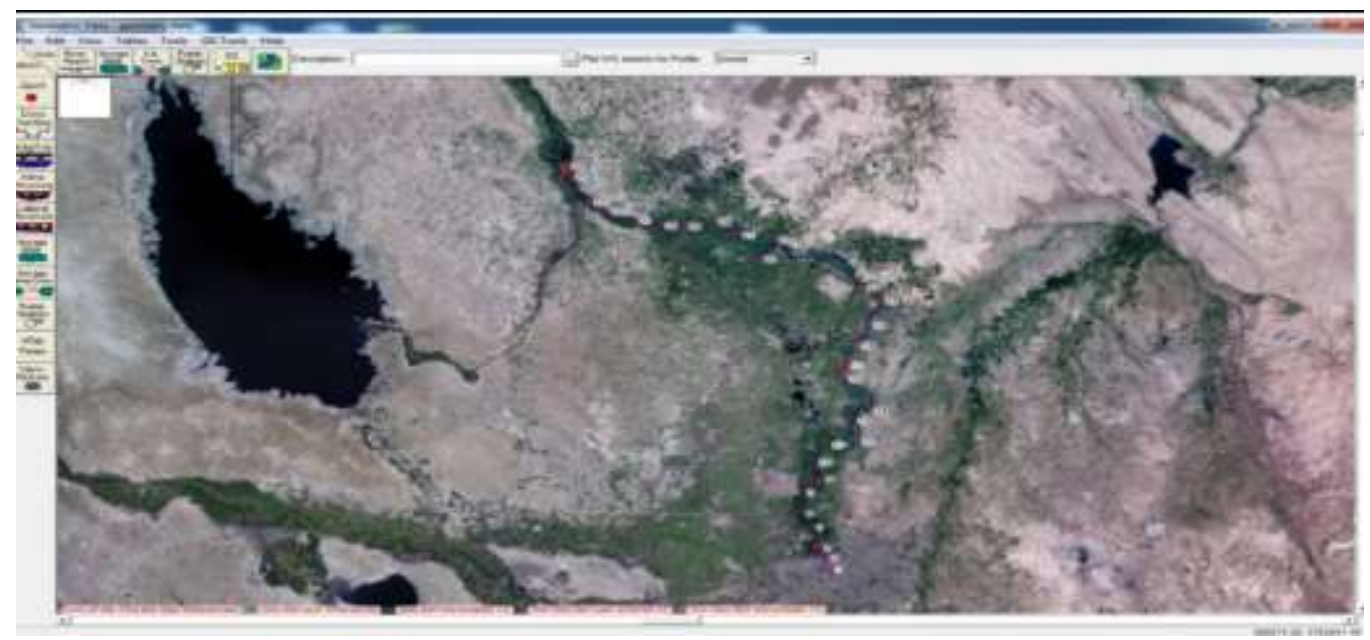

Figure 1: Tigris River in the Samarra barrage-to Baghdad city-Resafa station 


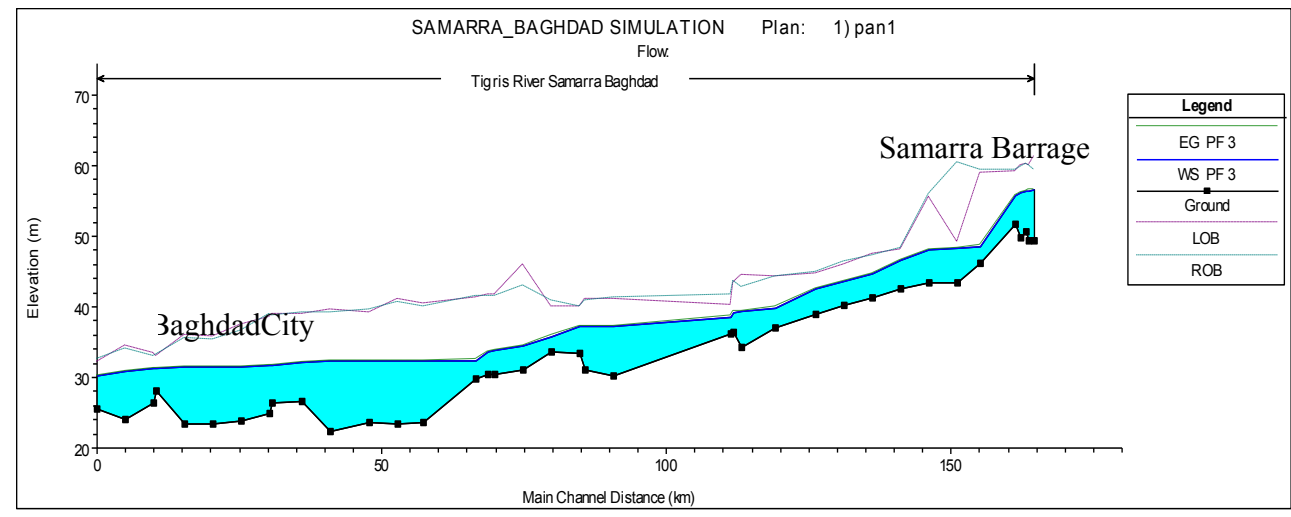

Figure 1 continued

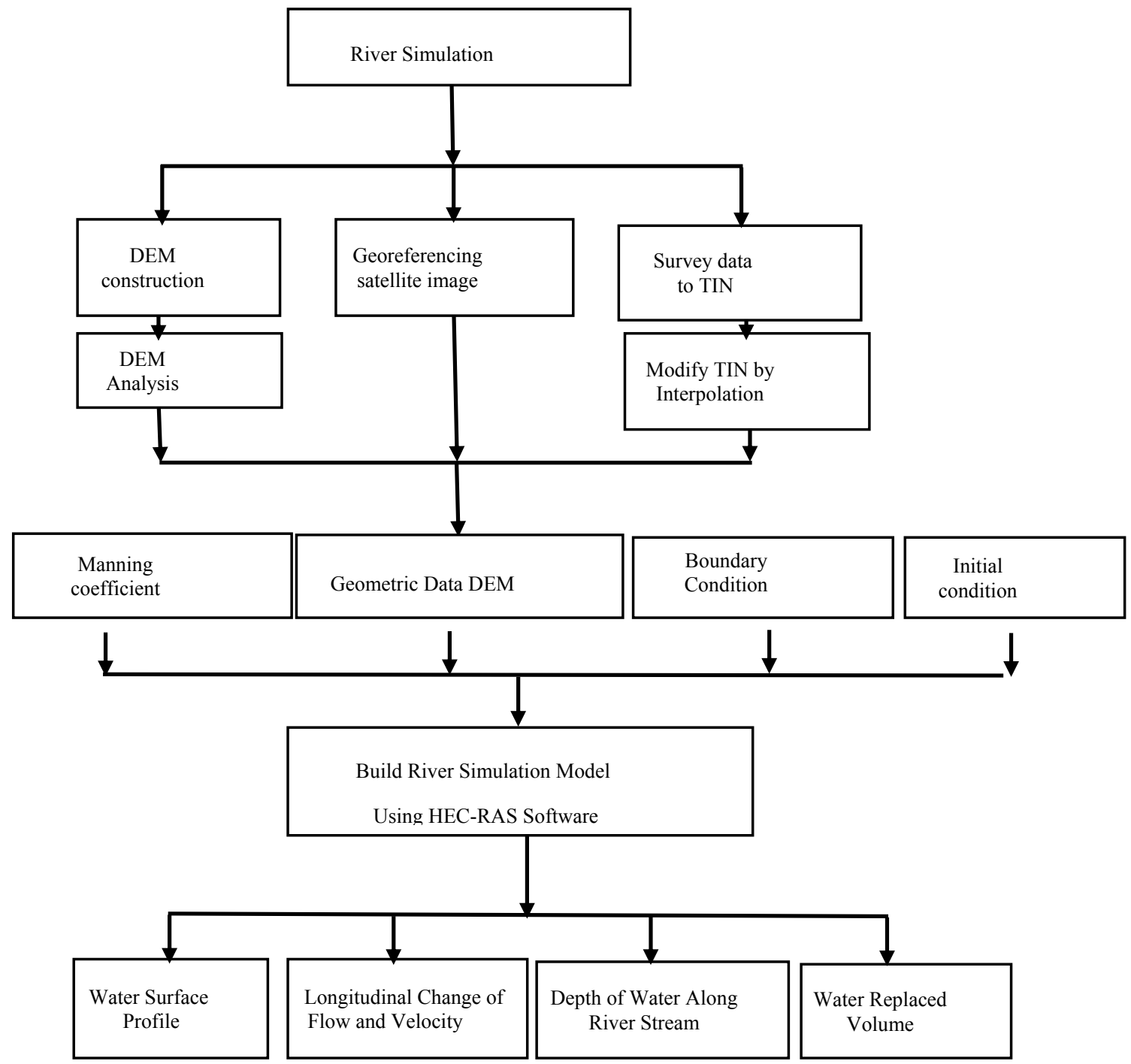

Figure 2: Methodology of Simulation

\subsection{Methodology}

The method used in this study is to obtain discharge and hydrological data from previous years. Hydraulic structures in the working route were determined (cross section areas, gradient between sections, hydraulic radius, manning coefficient). Then, HEC-RAS software was used to build simulates model the hydrodynamic flow and water flow within the river as shown at Figure (2). To obtain results that explain the reasons for the transformation of the Tigris River a swamp that collects pollutants and sediments that impede the movement of water.

\subsection{HEC-RAS Software}

HEC-RAS program is one of the 1D dynamic models developed by U.S. Army Corps. Hydrology makes calculations in semi unsteady, unsteady flows with one- and two-dimensional unsteady river flow calculations with one-dimensional steady 
hydraulic flow. The first version of HEC-RAS was released in July 1995.Since then many upgrades have been released from 1.1 to 5.07. In this study version 4.0 was used. In this program, subcritical, supercritical, and mixed flow solution options are provided for the determination of water surface profiles under stable current conditions. In this program, one-dimensional energy equation is used for the solutions made under steady flow conditions.

The physical laws in unsteady flow which govern the flow of water in a stream are:

(1) The principle of conservation of mass (continuity).

(2) The principle of conservation of momentum.

These laws are expressed mathematically in the form of partial differential equation, which will hereafter be referred to as continuity and momentum equations. The data required to run the program includes three groups that are entered in succession, as follows (19):

- Geometric Data:

- Initial Conditions

- Boundary Conditions

\subsection{Coefficient of determination $\boldsymbol{R}^{2}$}

It is square of the correlation (r) between observed and simulated values. $R^{2}$ Ranges from 0 to 1 mathematically, coefficient of determination $\left(R^{2}\right)$ is presented as :

$$
R^{2}=\left[\frac{\sum_{i=1}^{n}\left(Q_{i}^{o b s}-Q_{a v e}^{o b s}\right)\left(Q_{i}^{s i m}-Q_{a v e}^{s i m}\right)}{\left[\sum_{i=1}^{n}\left(Q_{i}^{o b s}-Q_{a v e}^{o b s}\right)^{2} \sum_{i=1}^{n}\left(Q_{i}^{s i m}-Q_{a v e}^{s i m}\right)^{2}\right]^{0.5}}\right]^{2}
$$

Where $Q_{i}^{o b s}$ isobservedvalue $\left(\mathrm{m}^{3} / \mathrm{s}\right), Q_{a v e}^{o b s}$ is the average observed value of n value, $Q_{i}^{s i m}$ is simulated value $\left(\mathrm{m}^{3} / s\right), Q_{a v e}^{s i m}$ is the average simulated value of $\mathrm{n}$ value and is the number of observation.

\section{Results}

Hydraulic analysis of the study was carried out using the HEC-RAS package program version of 4.0, Figure 3 (a, b). The data obtained because of the observations and measurement discharge, velocity, area, cross section, and Froude number of the state of flow in the Tigris River between the Samarra Dam and the city of Baghdad. The two scenarios (steady and unsteady).

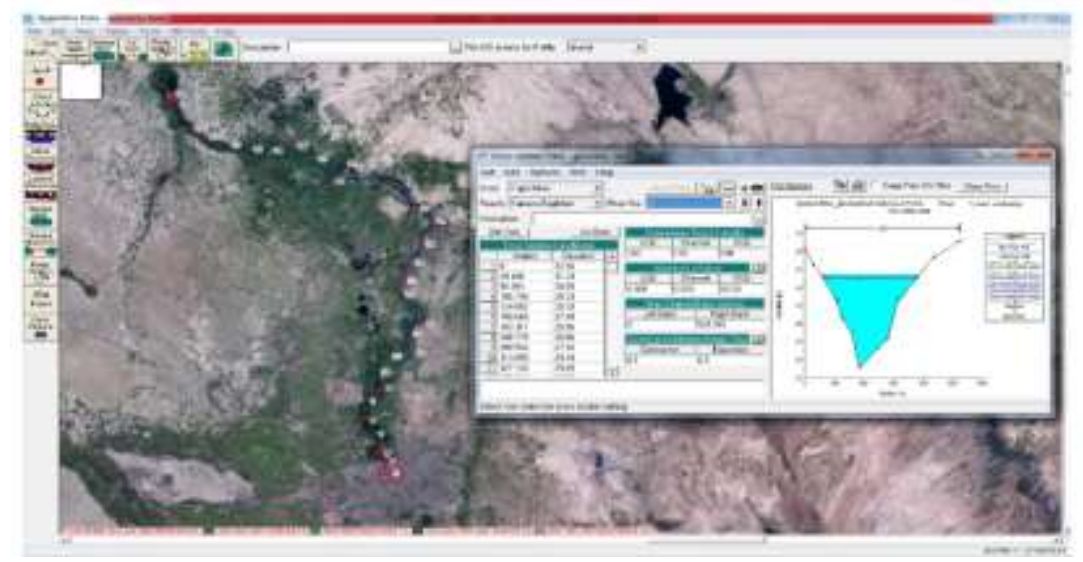

(a)

Figure 3: (a) Some forms of cross section of the Samara-Baghdad for the different station of the Tigris River section (1). (b) Some forms of cross section of the Samara-Baghdad for the different station of the Tigris River section (38)

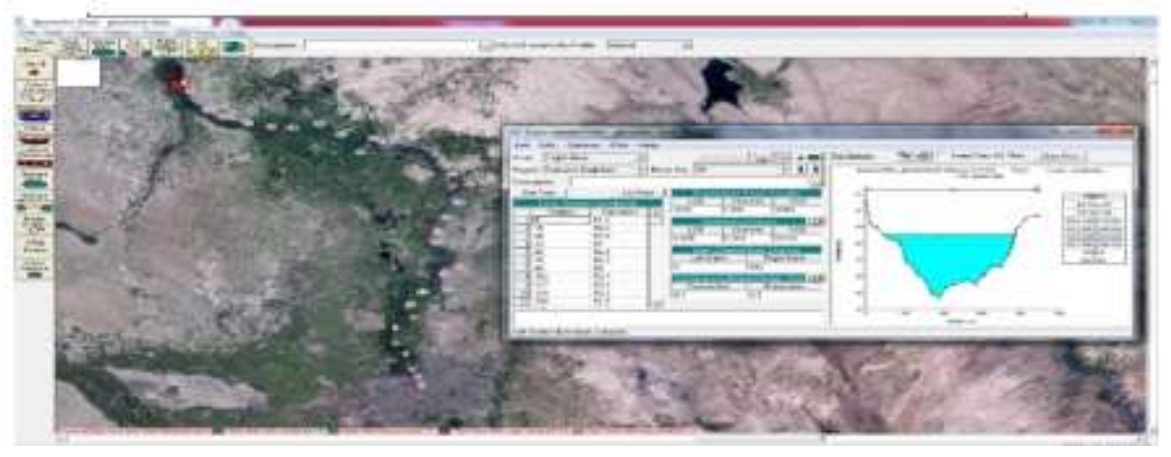

Figure 3 continued (b) 
1. First scenarios (steady): model has been operated to steady state based on the recorded discharge. The model operated for three possibilities (Max discharge, average discharge, and min discharge). The above results were checked where it was found that Max discharge reached $638.17 \mathrm{~m}^{3} / \mathrm{sec}$ at the section and reached the highest value of the flow velocity was within limits $2.12 \mathrm{~m} / \mathrm{sec}$ as for the highest area reached at $301.42 \mathrm{~m}^{2}$ at the section 15 show the Table (1) and Figure (4). When passing average discharge reached $557.86 \mathrm{~m}^{3} / \mathrm{sec}$ and the value of the flow velocity was limits $2.21 \mathrm{~m} / \mathrm{sec}$ as for the average area reached at $252.86 \mathrm{~m}^{2 \mathrm{v}}$ at the section 15 shows the Table (2) and Figure (5). When passing min discharge reached 504.5 $\mathrm{m}^{3} / \mathrm{sec}$ at the section and reached the min value of the flow velocity was within limit $0.13 \mathrm{~m} / \mathrm{sec}$ as for the min area reached at $4758.13 \mathrm{~m}^{2}$ at the section 11 , shown in Table (3) and Figure (6). The steady state successfully in Simulation results the velocity not more $2.5 \mathrm{~m} / \mathrm{sec}$ and Froude number not more 1.5 .

2. The second scenarios (unsteady): model has been operated to unsteady state based on the recorded discharge (20122017) years. The Max discharge reached $638.12 \mathrm{~m}^{3} / \mathrm{sec}$ at the section 34, The Min discharge reached $635.57 \mathrm{~m}^{3} / \mathrm{sec}$ at section (1). The Max velocity passing through the river reached $2.42 \mathrm{~m} / \mathrm{sec}$, the Min velocity passing through the river reached 0.14 $\mathrm{m} / \mathrm{sec}$. The Max Froude number reached 0.52 at section 34, the Min Froude number reached 0.02 at section 12 , as shown in Table (4).

Table 1: $\quad$ Monthly discharge of the city of Baghdad Sarai for the years 2012-2017 from (MOWR).

\begin{tabular}{ccccccc}
\hline Year & 2012 & 2013 & 2014 & 2015 & 2016 & 2017 \\
\hline Jan & 507 & 490 & 490 & 474 & 444 & 461 \\
Feb & 480 & 608 & 509 & 437 & 694 & 467 \\
Mar & 490 & 708 & 581 & 463 & 611 & 506 \\
Apr & 495 & 500 & 524 & 453 & 482 & 562 \\
May & 458 & 433 & 465 & 438 & 499 & 601 \\
Jun & 450 & 498 & 439 & 448 & 528 & 530 \\
Jul & 510 & 507 & 470 & 400 & 519 & 483 \\
Aug & 471 & 560 & 455 & 431 & 518 & 463 \\
Sep & 454 & 510 & 410 & 456 & 490 & 444 \\
Oct & 480 & 466 & 475 & 466 & 468 & 485 \\
Nov & 500 & 717 & 543 & 477 & 473 & 532 \\
Dec & 503 & 560 & 507 & 534 & 417 & 521 \\
\hline
\end{tabular}

Table 2: $\quad$ Monthly discharge of the city of Baghdad Sarai.

\begin{tabular}{ll}
\hline Calculate 2017 & Observed 2017 \\
\hline 467.4 & 416 \\
465.57 & 467 \\
524.19 & 506 \\
561.67 & 562 \\
604.55 & 601 \\
546.64 & 530 \\
480.98 & 483 \\
465.05 & 463 \\
415.13 & 444 \\
478.43 & 485 \\
506.07 & 532 \\
\hline
\end{tabular}

\subsection{Calibration}

HEC-RAS has the capabilities to process automated calibration to minimize a specific objective function, such as sum of the absolute error, sum of the squared error. Therefore, automated calibration in conjunction with manual calibration was used to determine a practical range of the parameter values preserving the hydrograph shape and minimum error in volumes. Flow calibration was carried out over the period 2012-2017 and model validation was carried out over the period of the same years in Baghdad Sarai as shown in Table (5). The coefficient of determination $\left(R^{2}\right)$ during calibration were found to be 0.906 . The model performance is reasonably good in simulating flows for periods of the calibration period. The simulated results achieved a success with an accuracy of $\mathrm{R}^{2} 0.906$, compared with the monthly discharge of the city of Baghdad Sarai for the years 20122017 from The Ministry of Water Resources (MOWR) [14]shown in the Table (5), Table(6) and Figure (4).

\section{Conclution}

There are several findings and outcomes that outlined from the investigation and analysis of this research, which can be summarized as follow:

1. The performance of the model was found to be rather good agreement with $R^{2}(0.9068)$ during the calibration periods.

2. The HEC-RAS can use successfully for modelling the ecosystem behaviour of Tigris River within a steady area. 
3. From the data result for steady- state model (maximum discharge, velocity, and minimum flow area) was 638.17 $\mathrm{m}^{3} / \mathrm{sec}, 2.12 \mathrm{~m} / \mathrm{sec}$ and $301.42 \mathrm{~m}^{2}$ respectively. It showed the weakness in the Tigris River (Samarra barrage-to Baghdad cityResafa station) these values occurred at station $66+700$. While, the minimum discharge, velocity and maximum flow area were $504.5 \mathrm{~m}^{3} / \mathrm{sec}, 0.13 \mathrm{~m} / \mathrm{sec}$ and $4758.13 \mathrm{~m}^{2}$ respectively. The results of unsteady state were analyzed based on the recorded discharge in the year 2018. The maximum and minimum discharge (flow velocity) were $638.12 \mathrm{~m}^{3} / \mathrm{sec}(2.42 \mathrm{~m} / \mathrm{sec})$ at the stations $94+666+7$ and $635.57 \mathrm{~m}^{3} / \mathrm{sec}(0.14 \mathrm{~m} / \mathrm{sec})$ at station $00+00$, respectively. The maximum and minimum Froude number were 0.52 and 0.02 at stations $94+666+7$ and $04+800$, respectively.

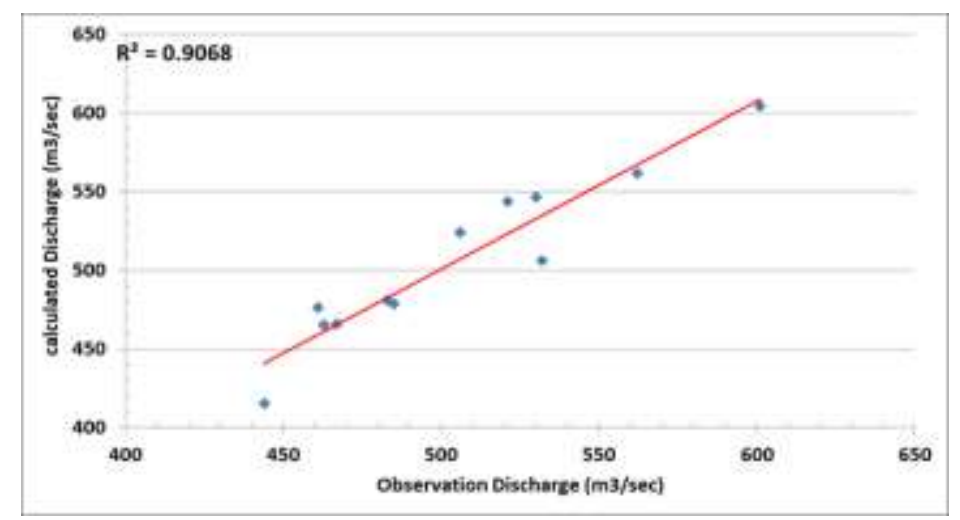

Figure 4: Scatter plot of calculated and observation discharge.

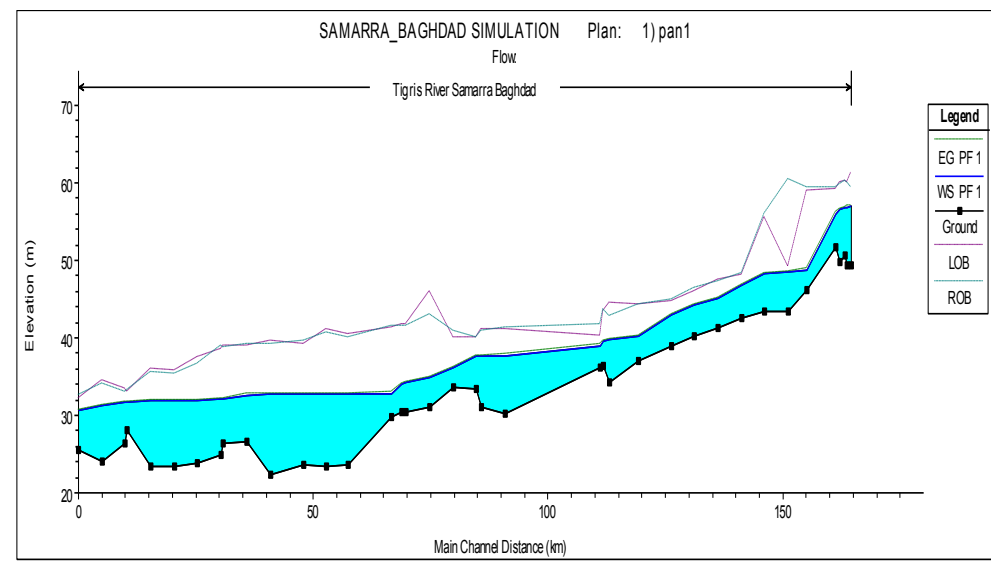

Figure 5: Reach in steady state with Mix discharge $(\mathrm{Q}=638.17 \mathrm{~m} 3 / \mathrm{sec})$.Appendix

Table 3: Output of the Tigris River of the steady stat Max discharge $(\mathrm{Q}=638.17 \mathrm{~m} 3 / \mathrm{sec})$

\begin{tabular}{|c|c|c|c|c|c|c|c|c|c|c|}
\hline Reach & $\begin{array}{c}\text { River } \\
\text { Sta }\end{array}$ & $\begin{array}{c}\mathrm{Q} \\
\text { Total } \\
\left(\mathrm{m}^{3} / \mathbf{s}\right)\end{array}$ & $\begin{array}{c}\text { Min } \\
\text { Ch El } \\
(m)\end{array}$ & $\begin{array}{c}\text { W.S. } \\
\text { Elev } \\
(m)\end{array}$ & $\begin{array}{l}\text { E.G. } \\
\text { Elev } \\
(m)\end{array}$ & $\begin{array}{c}\text { E.G. } \\
\text { Slope } \\
(\boldsymbol{m} / \boldsymbol{m})\end{array}$ & $\begin{array}{c}\text { Vel } \\
\text { Chnl } \\
(m / s)\end{array}$ & $\begin{array}{l}\text { Flow } \\
\text { Area } \\
\left(m^{2}\right)\end{array}$ & $\begin{array}{c}\text { Top } \\
\text { Width }\end{array}$ & $\begin{array}{c}\text { Froude } \\
\# \text { Chl }\end{array}$ \\
\hline $\begin{array}{l}\text { Samarra } \\
\text { Baghdad }\end{array}$ & 1 & 638.2 & 25.56 & 30.68 & 30.71 & 0.000142 & 0.78 & 814.5 & 326.09 & 0.16 \\
\hline $\begin{array}{l}\text { Samarra } \\
\text { Baghdad }\end{array}$ & 2 & 638.2 & 24.12 & 31.3 & 31.32 & 0.000106 & 0.65 & 984.05 & 419.1 & 0.14 \\
\hline $\begin{array}{l}\text { Samarra } \\
\text { Baghdad }\end{array}$ & 3 & 638.2 & 26.28 & 31.67 & 31.68 & 0.000054 & 0.43 & 1470.27 & 691.79 & 0.1 \\
\hline $\begin{array}{l}\text { Samarra } \\
\text { Baghdad }\end{array}$ & 4 & 638.2 & 28 & 31.7 & 31.71 & 0.000077 & 0.44 & 1446.36 & 861.51 & 0.11 \\
\hline $\begin{array}{l}\text { Samarra } \\
\text { Baghdad }\end{array}$ & 5 & 638.2 & 23.43 & 31.84 & 31.84 & 0.000012 & 0.24 & 2661.99 & 1009.14 & 0.05 \\
\hline $\begin{array}{l}\text { Samarra } \\
\text { Baghdad }\end{array}$ & 6 & 638.2 & 23.37 & 31.89 & 31.9 & 0.00001 & 0.23 & 2819.08 & 984.31 & 0.04 \\
\hline $\begin{array}{l}\text { Samarra } \\
\text { Baghdad }\end{array}$ & 7 & 638.2 & 23.84 & 31.96 & 31.97 & 0.000023 & 0.39 & 1640.22 & 477.66 & 0.07 \\
\hline $\begin{array}{l}\text { Samarra } \\
\text { Baghdad }\end{array}$ & 8 & 638.2 & 24.79 & 32.06 & 32.07 & 0.000016 & 0.37 & 1747.59 & 427.8 & 0.06 \\
\hline $\begin{array}{c}\text { Samarra } \\
\text { Baghdad }\end{array}$ & 9 & 638.2 & 26.28 & 32.07 & 32.08 & 0.000079 & 0.56 & 1133.94 & 481.08 & 0.12 \\
\hline $\begin{array}{c}\text { Samarra } \\
\text { Baghdad }\end{array}$ & 10 & 638.2 & 26.54 & 32.63 & 32.67 & 0.000186 & 0.92 & 692.68 & 266.12 & 0.18 \\
\hline $\begin{array}{l}\text { Samarra } \\
\text { Baghdad }\end{array}$ & 11 & 638.2 & 22.34 & 32.7 & 32.7 & 0.000002 & 0.13 & 4758.13 & 912.17 & 0.02 \\
\hline
\end{tabular}




\begin{tabular}{|c|c|c|c|c|c|c|c|c|c|c|}
\hline $\begin{array}{l}\text { Samarra } \\
\text { Baghdad }\end{array}$ & 12 & 638.2 & 23.54 & 32.72 & 32.72 & 0.000003 & 0.16 & 4033.48 & 963.2 & 0.02 \\
\hline $\begin{array}{l}\text { Samarra } \\
\text { Baghdad }\end{array}$ & 13 & 638.2 & 23.45 & 32.73 & 32.73 & 0.000002 & 0.15 & 4279.19 & 755.72 & 0.02 \\
\hline $\begin{array}{l}\text { Samarra } \\
\text { Baghdad }\end{array}$ & 14 & 638.2 & 23.66 & 32.74 & 32.74 & 0.000007 & 0.36 & 1762.23 & 239.43 & 0.04 \\
\hline $\begin{array}{l}\text { Samarra } \\
\text { Baghdad }\end{array}$ & 15 & 638.2 & 29.85 & 32.82 & 33.05 & 0.001586 & 2.12 & 301.42 & 165.75 & 0.5 \\
\hline $\begin{array}{l}\text { Samarra } \\
\text { Baghdad }\end{array}$ & 16 & 638.2 & 30.46 & 34 & 34.04 & 0.000206 & 0.9 & 711.34 & 306.56 & 0.19 \\
\hline $\begin{array}{l}\text { Samarra } \\
\text { Baghdad }\end{array}$ & 17 & 638.2 & 30.46 & 34.19 & 34.23 & 0.00016 & 0.83 & 768.87 & 308.03 & 0.17 \\
\hline $\begin{array}{l}\text { Samarra } \\
\text { Baghdad }\end{array}$ & 18 & 638.2 & 31.14 & 34.88 & 34.9 & 0.000116 & 0.71 & 901.44 & 359.32 & 0.14 \\
\hline $\begin{array}{l}\text { Samarra } \\
\text { Baghdad }\end{array}$ & 19 & 638.2 & 33.54 & 36.09 & 36.21 & 0.00095 & 1.48 & 430.72 & 275.43 & 0.38 \\
\hline $\begin{array}{l}\text { Samarra } \\
\text { Baghdad }\end{array}$ & 20 & 638.2 & 33.34 & 37.58 & 37.61 & 0.000132 & 0.8 & 802.15 & 294.19 & 0.15 \\
\hline $\begin{array}{l}\text { Samarra } \\
\text { Baghdad }\end{array}$ & 21 & 638.2 & 31.12 & 37.65 & 37.66 & 0.000025 & 0.48 & 1335.51 & 295.43 & 0.07 \\
\hline $\begin{array}{l}\text { Samarra } \\
\text { Baghdad }\end{array}$ & 22 & 638.2 & 30.12 & 37.76 & 37.77 & 0.000019 & 0.35 & 1831.98 & 544.11 & 0.06 \\
\hline $\begin{array}{l}\text { Samarra } \\
\text { Baghdad }\end{array}$ & 23 & 638.2 & 36.26 & 38.89 & 39.06 & 0.001384 & 1.85 & 351.27 & 224.43 & 0.46 \\
\hline $\begin{array}{l}\text { Samarra } \\
\text { Baghdad }\end{array}$ & 24 & 638.2 & 36.36 & 39.5 & 39.61 & 0.000856 & 1.46 & 438.12 & 265.86 & 0.36 \\
\hline $\begin{array}{l}\text { Samarra } \\
\text { Baghdad }\end{array}$ & 25 & 638.2 & 34.23 & 39.74 & 39.75 & 0.00003 & 0.45 & 1430.07 & 416.09 & 0.08 \\
\hline $\begin{array}{l}\text { Samarra } \\
\text { Baghdad }\end{array}$ & 26 & 638.2 & 37.11 & 40.18 & 40.26 & 0.00063 & 1.27 & 501.85 & 296.95 & 0.31 \\
\hline $\begin{array}{l}\text { Samarra } \\
\text { Baghdad }\end{array}$ & 27 & 638.2 & 39 & 43.02 & 43.06 & 0.000275 & 0.85 & 746.92 & 430.59 & 0.21 \\
\hline $\begin{array}{l}\text { Samarra } \\
\text { Baghdad }\end{array}$ & 28 & 638.2 & 40.22 & 44.16 & 44.18 & 0.000185 & 0.58 & 1097.07 & 838.21 & 0.16 \\
\hline $\begin{array}{c}\text { Samarra } \\
\text { Baghdad }\end{array}$ & 29 & 638.2 & 41.37 & 45.14 & 45.17 & 0.000212 & 0.76 & 842.91 & 479.8 & 0.18 \\
\hline $\begin{array}{l}\text { Samarra } \\
\text { Baghdad }\end{array}$ & 30 & 638.2 & 42.45 & 46.78 & 46.82 & 0.000577 & 0.86 & 741.69 & 737.83 & 0.27 \\
\hline $\begin{array}{l}\text { Samarra } \\
\text { Baghdad }\end{array}$ & 31 & 638.2 & 43.3 & 48.32 & 48.36 & 0.000191 & 0.81 & 790.92 & 374.61 & 0.18 \\
\hline $\begin{array}{l}\text { Samarra } \\
\text { Baghdad }\end{array}$ & 32 & 638.2 & 43.3 & 48.61 & 48.61 & 0.000023 & 0.33 & 1956.81 & 738.25 & 0.06 \\
\hline $\begin{array}{l}\text { Samarra } \\
\text { Baghdad }\end{array}$ & 33 & 638.2 & 46.1 & 48.79 & 48.95 & 0.001919 & 1.8 & 355.4 & 288.93 & 0.52 \\
\hline $\begin{array}{l}\text { Samarra } \\
\text { Baghdad }\end{array}$ & 34 & 638.2 & 51.7 & 55.91 & 56.09 & 0.000808 & 1.9 & 336.42 & 131.18 & 0.38 \\
\hline $\begin{array}{l}\text { Samarra } \\
\text { Baghdad }\end{array}$ & 35 & 638.2 & 49.8 & 56.51 & 56.61 & 0.000344 & 1.41 & 453.6 & 145.56 & 0.25 \\
\hline $\begin{array}{l}\text { Samarra } \\
\text { Baghdad }\end{array}$ & 36 & 638.2 & 50.6 & 56.8 & 56.85 & 0.000177 & 1.05 & 609.68 & 184.56 & 0.18 \\
\hline $\begin{array}{l}\text { Samarra } \\
\text { Baghdad }\end{array}$ & 37 & 638.2 & 49.4 & 56.88 & 56.93 & 0.000148 & 1 & 639.68 & 182.56 & 0.17 \\
\hline $\begin{array}{l}\text { Samarra } \\
\text { Baghdad }\end{array}$ & 38 & 638.2 & 49.4 & 56.97 & 56.98 & 0.000022 & 0.44 & 1462.55 & 341.24 & 0.07 \\
\hline
\end{tabular}

Table 4: Output of the Tigris River of the steady stat for Average Discharge $(\mathrm{Q}=557.86 \mathrm{~m} 3 / \mathrm{sec})$

\begin{tabular}{|c|c|c|c|c|c|c|c|c|c|c|}
\hline Reach & $\begin{array}{l}\text { River } \\
\text { Sta }\end{array}$ & $\begin{array}{c}\text { Q } \\
\text { Total }\end{array}$ & $\begin{array}{c}\text { Min } \\
\text { Ch El }\end{array}$ & $\begin{array}{l}\text { W.S. } \\
\text { Elev }\end{array}$ & $\begin{array}{l}\text { E.G. } \\
\text { Elev }\end{array}$ & $\begin{array}{l}\text { E.G. } \\
\text { Slope }\end{array}$ & $\begin{array}{c}\text { Vel } \\
\text { Chnl }\end{array}$ & $\begin{array}{l}\text { Flow } \\
\text { Area }\end{array}$ & $\begin{array}{c}\text { Top } \\
\text { Width }\end{array}$ & $\begin{array}{c}\text { Froude } \\
\# \text { Chl }\end{array}$ \\
\hline & & $(m 3 / s)$ & $(m)$ & $(m)$ & $(m)$ & $(m / m)$ & $(m / s)$ & $(m 2)$ & (m) & \\
\hline $\begin{array}{l}\text { Samarra } \\
\text { Baghdad }\end{array}$ & 1 & 557.86 & 25.56 & 30.41 & 30.44 & 0.000142 & 0.76 & 729.86 & 303.19 & 0.16 \\
\hline $\begin{array}{l}\text { Samarra } \\
\text { Baghdad }\end{array}$ & 2 & 557.86 & 24.12 & 31.04 & 31.06 & 0.00011 & 0.64 & 878.21 & 395.27 & 0.14 \\
\hline $\begin{array}{l}\text { Samarra } \\
\text { Baghdad }\end{array}$ & 3 & 557.86 & 26.28 & 31.44 & 31.44 & 0.000059 & 0.43 & 1308.59 & 672.31 & 0.1 \\
\hline $\begin{array}{l}\text { Samarra } \\
\text { Baghdad }\end{array}$ & 4 & 557.86 & 28 & 31.47 & 31.48 & 0.000093 & 0.45 & 1247.6 & 845.25 & 0.12 \\
\hline $\begin{array}{l}\text { Samarra } \\
\text { Baghdad }\end{array}$ & 5 & 557.86 & 23.43 & 31.61 & 31.62 & 0.000013 & 0.23 & 2437.05 & 999.32 & 0.05 \\
\hline $\begin{array}{l}\text { Samarra } \\
\text { Baghdad }\end{array}$ & 6 & 557.86 & 23.37 & 31.67 & 31.67 & 0.00001 & 0.21 & 2599.33 & 979.02 & 0.04 \\
\hline $\begin{array}{l}\text { Samarra } \\
\text { Baghdad }\end{array}$ & 7 & 557.86 & 23.84 & 31.74 & 31.74 & 0.000021 & 0.36 & 1533.79 & 464.42 & 0.06 \\
\hline
\end{tabular}




\begin{tabular}{|c|c|c|c|c|c|c|c|c|c|c|}
\hline $\begin{array}{l}\text { Samarra } \\
\text { Baghdad }\end{array}$ & 8 & 557.86 & 24.79 & 31.82 & 31.83 & 0.000014 & 0.34 & 1648.58 & 414.09 & 0.05 \\
\hline $\begin{array}{l}\text { Samarra } \\
\text { Baghdad }\end{array}$ & 9 & 557.86 & 26.28 & 31.83 & 31.85 & 0.000078 & 0.54 & 1023.96 & 449.14 & 0.12 \\
\hline $\begin{array}{l}\text { Samarra } \\
\text { Baghdad }\end{array}$ & 10 & 557.86 & 26.54 & 32.38 & 32.42 & 0.000178 & 0.89 & 628.11 & 246.99 & 0.18 \\
\hline $\begin{array}{l}\text { Samarra } \\
\text { Baghdad }\end{array}$ & 11 & 557.86 & 22.34 & 32.44 & 32.44 & 0.000001 & 0.12 & 4523.91 & 907.41 & 0.02 \\
\hline $\begin{array}{l}\text { Samarra } \\
\text { Baghdad }\end{array}$ & 12 & 557.86 & 23.54 & 32.46 & 32.46 & 0.000003 & 0.15 & 3784.68 & 960.6 & 0.02 \\
\hline $\begin{array}{l}\text { Samarra } \\
\text { Baghdad }\end{array}$ & 13 & 557.86 & 23.45 & 32.47 & 32.47 & 0.000002 & 0.14 & 4083.55 & 751.33 & 0.02 \\
\hline $\begin{array}{l}\text { Samarra } \\
\text { Baghdad }\end{array}$ & 14 & 557.86 & 23.66 & 32.48 & 32.48 & 0.000006 & 0.33 & 1699.96 & 238.38 & 0.04 \\
\hline $\begin{array}{l}\text { Samarra } \\
\text { Baghdad }\end{array}$ & 15 & 557.86 & 29.85 & 32.51 & 32.76 & 0.001948 & 2.21 & 252.86 & 152.53 & 0.55 \\
\hline $\begin{array}{l}\text { Samarra } \\
\text { Baghdad }\end{array}$ & 16 & 557.86 & 30.46 & 33.8 & 33.84 & 0.000212 & 0.86 & 649.12 & 304.97 & 0.19 \\
\hline $\begin{array}{l}\text { Samarra } \\
\text { Baghdad }\end{array}$ & 17 & 557.86 & 30.46 & 33.99 & 34.02 & 0.00016 & 0.79 & 707.21 & 306.46 & 0.17 \\
\hline $\begin{array}{l}\text { Samarra } \\
\text { Baghdad }\end{array}$ & 18 & 557.86 & 31.14 & 34.67 & 34.7 & 0.000115 & 0.67 & 828.14 & 353.15 & 0.14 \\
\hline $\begin{array}{l}\text { Samarra } \\
\text { Baghdad }\end{array}$ & 19 & 557.86 & 33.54 & 35.91 & 36.02 & 0.001054 & 1.46 & 381.42 & 269.1 & 0.39 \\
\hline $\begin{array}{l}\text { Samarra } \\
\text { Baghdad }\end{array}$ & 20 & 557.86 & 33.34 & 37.35 & 37.38 & 0.000121 & 0.76 & 736.92 & 273.74 & 0.15 \\
\hline $\begin{array}{l}\text { Samarra } \\
\text { Baghdad }\end{array}$ & 21 & 557.86 & 31.12 & 37.42 & 37.43 & 0.000023 & 0.44 & 1267 & 294.56 & 0.07 \\
\hline $\begin{array}{l}\text { Samarra } \\
\text { Baghdad }\end{array}$ & 22 & 557.86 & 30.12 & 37.53 & 37.53 & 0.000019 & 0.33 & 1701.6 & 544.09 & 0.06 \\
\hline $\begin{array}{l}\text { Samarra } \\
\text { Baghdad }\end{array}$ & 23 & 557.86 & 36.26 & 38.65 & 38.83 & 0.001759 & 1.91 & 296.99 & 217.5 & 0.51 \\
\hline $\begin{array}{l}\text { Samarra } \\
\text { Baghdad }\end{array}$ & 24 & 557.86 & 36.36 & 39.35 & 39.45 & 0.00089 & 1.4 & 397.66 & 262.86 & 0.36 \\
\hline $\begin{array}{l}\text { Samarra } \\
\text { Baghdad }\end{array}$ & 25 & 557.86 & 34.23 & 39.57 & 39.57 & 0.000027 & 0.41 & 1359.26 & 412.15 & 0.07 \\
\hline $\begin{array}{l}\text { Samarra } \\
\text { Baghdad }\end{array}$ & 26 & 557.86 & 37.11 & 39.97 & 40.05 & 0.000682 & 1.26 & 441.62 & 280.04 & 0.32 \\
\hline $\begin{array}{l}\text { Samarra } \\
\text { Baghdad }\end{array}$ & 27 & 557.86 & 39 & 42.78 & 42.82 & 0.000257 & 0.85 & 653.25 & 357.77 & 0.2 \\
\hline $\begin{array}{c}\text { Samarra } \\
\text { Baghdad }\end{array}$ & 28 & 557.86 & 40.22 & 43.87 & 43.89 & 0.000183 & 0.63 & 888.11 & 598.45 & 0.16 \\
\hline $\begin{array}{l}\text { Samarra } \\
\text { Baghdad }\end{array}$ & 29 & 557.86 & 41.37 & 44.89 & 44.92 & 0.000233 & 0.77 & 729.1 & 437.81 & 0.19 \\
\hline $\begin{array}{l}\text { Samarra } \\
\text { Baghdad }\end{array}$ & 30 & 557.86 & 42.45 & 46.67 & 46.71 & 0.000609 & 0.84 & 661.93 & 707.45 & 0.28 \\
\hline $\begin{array}{l}\text { Samarra } \\
\text { Baghdad }\end{array}$ & 31 & 557.86 & 43.3 & 48.19 & 48.22 & 0.000181 & 0.75 & 741.34 & 374.6 & 0.17 \\
\hline $\begin{array}{l}\text { Samarra } \\
\text { Baghdad }\end{array}$ & 32 & 557.86 & 43.3 & 48.45 & 48.46 & 0.000021 & 0.3 & 1843.73 & 735.16 & 0.06 \\
\hline $\begin{array}{l}\text { Samarra } \\
\text { Baghdad }\end{array}$ & 33 & 557.86 & 46.1 & 48.62 & 48.79 & 0.002259 & 1.82 & 306.22 & 275.36 & 0.55 \\
\hline $\begin{array}{l}\text { Samarra } \\
\text { Baghdad }\end{array}$ & 34 & 557.86 & 51.7 & 55.77 & 55.92 & 0.000733 & 1.76 & 317.55 & 129.06 & 0.36 \\
\hline $\begin{array}{l}\text { Samarra } \\
\text { Baghdad }\end{array}$ & 35 & 557.86 & 49.8 & 56.31 & 56.4 & 0.000321 & 1.31 & 424.87 & 143.57 & 0.24 \\
\hline $\begin{array}{l}\text { Samarra } \\
\text { Baghdad }\end{array}$ & 36 & 557.86 & 50.6 & 56.57 & 56.62 & 0.000163 & 0.98 & 569.14 & 178.86 & 0.18 \\
\hline $\begin{array}{l}\text { Samarra } \\
\text { Baghdad }\end{array}$ & 37 & 557.86 & 49.4 & 56.65 & 56.7 & 0.000136 & 0.93 & 598.32 & 176.87 & 0.16 \\
\hline $\begin{array}{l}\text { Samarra } \\
\text { Baghdad }\end{array}$ & 38 & 557.86 & 49.4 & 56.73 & 56.74 & 0.000019 & 0.4 & 1381.54 & 333.53 & 0.06 \\
\hline
\end{tabular}




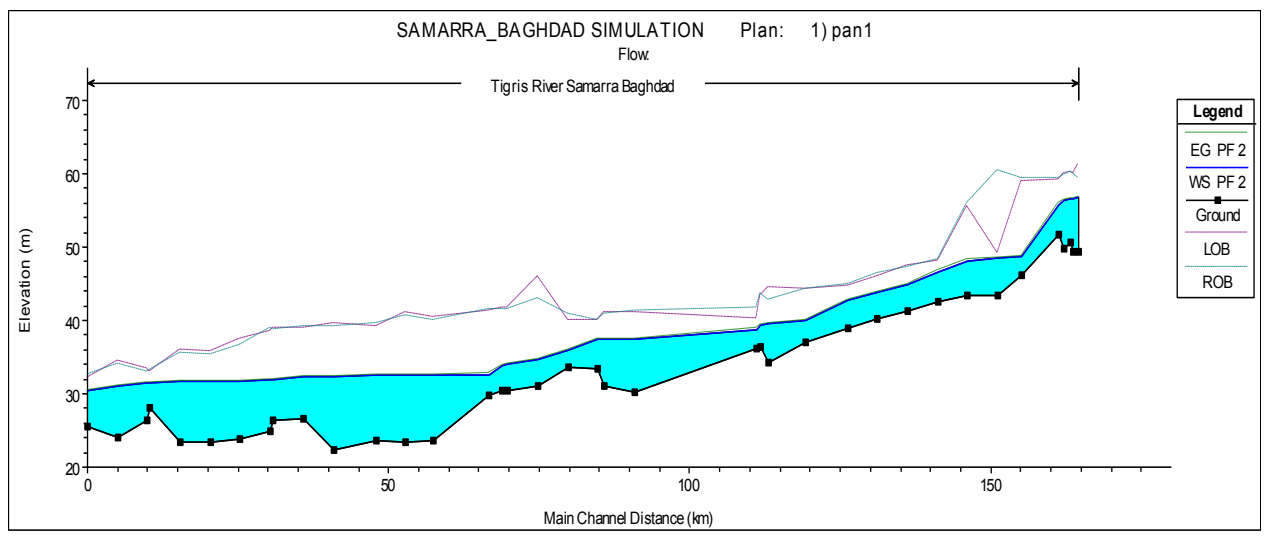

Figure 6: Reach in steady state with average discharge $(\mathrm{Q}=557.86 \mathrm{~m} 3 / \mathrm{sec})$

Table 5: Output of the Tigris River of the steady stat for Min Discharge ( $\mathrm{Q}=504.5 \mathrm{~m} 3 / \mathrm{sec})$

\begin{tabular}{|c|c|c|c|c|c|c|c|c|c|c|}
\hline Reach & $\begin{array}{c}\text { River } \\
\text { Sta }\end{array}$ & $\begin{array}{c}\text { Q } \\
\text { Total } \\
(\mathbf{m} 3 / \mathbf{s})\end{array}$ & $\begin{array}{c}\text { Min } \\
\text { Ch El } \\
(m)\end{array}$ & $\begin{array}{l}\text { W.S. } \\
\text { Elev } \\
(m)\end{array}$ & $\begin{array}{c}\text { E.G. } \\
\text { Elev } \\
(\boldsymbol{m})\end{array}$ & $\begin{array}{c}\text { E.G. } \\
\text { Slope } \\
(\boldsymbol{m} / \boldsymbol{m})\end{array}$ & $\begin{array}{c}\text { Vel } \\
\text { Chnl } \\
(\boldsymbol{m} / \mathbf{s})\end{array}$ & $\begin{array}{l}\text { Flow } \\
\text { Area } \\
(m 2)\end{array}$ & $\begin{array}{c}\text { Top } \\
\text { Width }\end{array}$ & $\begin{array}{c}\text { Froude } \\
\# \text { Chl }\end{array}$ \\
\hline $\begin{array}{l}\text { Samarra } \\
\text { Baghdad }\end{array}$ & 38 & 638.17 & 49.4 & 56.97 & 56.98 & $\begin{array}{c}2.20 \mathrm{E}- \\
05\end{array}$ & 0.44 & 1462.55 & 341.24 & 0.07 \\
\hline $\begin{array}{l}\text { Samarra } \\
\text { Baghdad }\end{array}$ & 1 & 638.17 & 25.56 & 30.68 & 30.71 & 0.00014 & 0.78 & 814.5 & 326.09 & 0.16 \\
\hline $\begin{array}{l}\text { Samarra } \\
\text { Baghdad }\end{array}$ & 2 & 638.17 & 24.12 & 31.3 & 31.32 & 0.00011 & 0.65 & 984.05 & 419.1 & 0.14 \\
\hline $\begin{array}{l}\text { Samarra } \\
\text { Baghdad }\end{array}$ & 3 & 638.17 & 26.28 & 31.67 & 31.68 & $\begin{array}{c}5.40 \mathrm{E}- \\
05\end{array}$ & 0.43 & 1470.27 & 691.79 & 0.1 \\
\hline $\begin{array}{l}\text { Samarra } \\
\text { Baghdad }\end{array}$ & 4 & 638.17 & 28 & 31.7 & 31.71 & $\begin{array}{c}7.70 \mathrm{E}- \\
05\end{array}$ & 0.44 & 1446.36 & 861.51 & 0.11 \\
\hline $\begin{array}{l}\text { Samarra } \\
\text { Baghdad }\end{array}$ & 5 & 638.17 & 23.43 & 31.84 & 31.84 & $\begin{array}{c}1.20 \mathrm{E}- \\
05\end{array}$ & 0.24 & 2661.99 & 1009.14 & 0.05 \\
\hline $\begin{array}{l}\text { Samarra } \\
\text { Baghdad }\end{array}$ & 6 & 638.17 & 23.37 & 31.89 & 31.9 & 0.00001 & 0.23 & 2819.08 & 984.31 & 0.04 \\
\hline $\begin{array}{l}\text { Samarra } \\
\text { Baghdad }\end{array}$ & 7 & 638.17 & 23.84 & 31.96 & 31.97 & $\begin{array}{c}2.30 \mathrm{E}- \\
05\end{array}$ & 0.39 & 1640.22 & 477.66 & 0.07 \\
\hline $\begin{array}{l}\text { Samarra } \\
\text { Baghdad }\end{array}$ & 8 & 638.17 & 24.79 & 32.06 & 32.07 & $\begin{array}{c}1.60 \mathrm{E}- \\
05\end{array}$ & 0.37 & 1747.59 & 427.8 & 0.06 \\
\hline $\begin{array}{l}\text { Samarra } \\
\text { Baghdad }\end{array}$ & 9 & 638.17 & 26.28 & 32.07 & 32.08 & $\begin{array}{c}7.90 \mathrm{E}- \\
05\end{array}$ & 0.56 & 1133.94 & 481.08 & 0.12 \\
\hline $\begin{array}{l}\text { Samarra } \\
\text { Baghdad }\end{array}$ & 10 & 638.17 & 26.54 & 32.63 & 32.67 & 0.00019 & 0.92 & 692.68 & 266.12 & 0.18 \\
\hline $\begin{array}{l}\text { Samarra } \\
\text { Baghdad }\end{array}$ & 11 & 638.17 & 22.34 & 32.7 & 32.7 & $\begin{array}{c}2.00 \mathrm{E}- \\
06\end{array}$ & 0.13 & 4758.13 & 912.17 & 0.02 \\
\hline $\begin{array}{l}\text { Samarra } \\
\text { Baghdad }\end{array}$ & 12 & 638.17 & 23.54 & 32.72 & 32.72 & $\begin{array}{c}3.00 \mathrm{E}- \\
06\end{array}$ & 0.16 & 4033.48 & 963.2 & 0.02 \\
\hline $\begin{array}{l}\text { Samarra } \\
\text { Baghdad }\end{array}$ & 13 & 638.17 & 23.45 & 32.73 & 32.73 & $\begin{array}{c}2.00 \mathrm{E}- \\
06\end{array}$ & 0.15 & 4279.19 & 755.72 & 0.02 \\
\hline $\begin{array}{l}\text { Samarra } \\
\text { Baghdad }\end{array}$ & 14 & 638.17 & 23.66 & 32.74 & 32.74 & $\begin{array}{c}7.00 \mathrm{E}- \\
06\end{array}$ & 0.36 & 1762.23 & 239.43 & 0.04 \\
\hline $\begin{array}{l}\text { Samarra } \\
\text { Baghdad }\end{array}$ & 15 & 638.17 & 29.85 & 32.82 & 33.05 & 0.00159 & 2.12 & 301.42 & 165.75 & 0.5 \\
\hline $\begin{array}{l}\text { Samarra } \\
\text { Baghdad }\end{array}$ & 16 & 638.17 & 30.46 & 34 & 34.04 & 0.00021 & 0.9 & 711.34 & 306.56 & 0.19 \\
\hline $\begin{array}{l}\text { Samarra } \\
\text { Baghdad }\end{array}$ & 17 & 638.17 & 30.46 & 34.19 & 34.23 & 0.00016 & 0.83 & 768.87 & 308.03 & 0.17 \\
\hline $\begin{array}{l}\text { Samarra } \\
\text { Baghdad }\end{array}$ & 18 & 638.17 & 31.14 & 34.88 & 34.9 & 0.00012 & 0.71 & 901.44 & 359.32 & 0.14 \\
\hline $\begin{array}{l}\text { Samarra } \\
\text { Baghdad }\end{array}$ & 19 & 638.17 & 33.54 & 36.09 & 36.21 & 0.00095 & 1.48 & 430.72 & 275.43 & 0.38 \\
\hline $\begin{array}{l}\text { Samarra } \\
\text { Baghdad }\end{array}$ & 20 & 638.17 & 33.34 & 37.58 & 37.61 & 0.00013 & 0.8 & 802.15 & 294.19 & 0.15 \\
\hline $\begin{array}{l}\text { Samarra } \\
\text { Baghdad }\end{array}$ & 21 & 638.17 & 31.12 & 37.65 & 37.66 & $\begin{array}{c}2.50 \mathrm{E}- \\
05\end{array}$ & 0.48 & 1335.51 & 295.43 & 0.07 \\
\hline $\begin{array}{l}\text { Samarra } \\
\text { Baghdad }\end{array}$ & 22 & 638.17 & 30.12 & 37.76 & 37.77 & $\begin{array}{c}1.90 \mathrm{E}- \\
05\end{array}$ & 0.35 & 1831.98 & 544.11 & 0.06 \\
\hline $\begin{array}{l}\text { Samarra } \\
\text { Baghdad }\end{array}$ & 23 & 638.17 & 36.26 & 38.89 & 39.06 & 0.00138 & 1.85 & 351.27 & 224.43 & 0.46 \\
\hline $\begin{array}{l}\text { Samarra } \\
\text { Baghdad }\end{array}$ & 24 & 638.17 & 36.36 & 39.5 & 39.61 & 0.00086 & 1.46 & 438.12 & 265.86 & 0.36 \\
\hline
\end{tabular}




\begin{tabular}{|c|c|c|c|c|c|c|c|c|c|c|}
\hline $\begin{array}{l}\text { Samarra } \\
\text { Baghdad }\end{array}$ & 25 & 638.17 & 34.23 & 39.74 & 39.75 & 0.00003 & 0.45 & 1430.07 & 416.09 & 0.08 \\
\hline $\begin{array}{l}\text { Samarra } \\
\text { Baghdad }\end{array}$ & 26 & 638.17 & 37.11 & 40.18 & 40.26 & 0.00063 & 1.27 & 501.85 & 296.95 & 0.31 \\
\hline $\begin{array}{l}\text { Samarra } \\
\text { Baghdad }\end{array}$ & 27 & 638.17 & 39 & 43.02 & 43.06 & 0.00028 & 0.85 & 746.92 & 430.59 & 0.21 \\
\hline $\begin{array}{l}\text { Samarra } \\
\text { Baghdad }\end{array}$ & 28 & 638.17 & 40.22 & 44.16 & 44.18 & 0.00019 & 0.58 & 1097.07 & 838.21 & 0.16 \\
\hline $\begin{array}{l}\text { Samarra } \\
\text { Baghdad }\end{array}$ & 29 & 638.17 & 41.37 & 45.14 & 45.17 & 0.00021 & 0.76 & 842.91 & 479.8 & 0.18 \\
\hline $\begin{array}{l}\text { Samarra } \\
\text { Baghdad }\end{array}$ & 30 & 638.17 & 42.45 & 46.78 & 46.82 & 0.00058 & 0.86 & 741.69 & 737.83 & 0.27 \\
\hline $\begin{array}{l}\text { Samarra } \\
\text { Baghdad }\end{array}$ & 31 & 638.17 & 43.3 & 48.32 & 48.36 & 0.00019 & 0.81 & 790.92 & 374.61 & 0.18 \\
\hline $\begin{array}{l}\text { Samarra } \\
\text { Baghdad }\end{array}$ & 32 & 638.17 & 43.3 & 48.61 & 48.61 & $\begin{array}{c}2.30 \mathrm{E}- \\
05\end{array}$ & 0.33 & 1956.81 & 738.25 & 0.06 \\
\hline $\begin{array}{l}\text { Samarra } \\
\text { Baghdad }\end{array}$ & 33 & 638.17 & 46.1 & 48.79 & 48.95 & 0.00192 & 1.8 & 355.4 & 288.93 & 0.52 \\
\hline $\begin{array}{l}\text { Samarra } \\
\text { Baghdad }\end{array}$ & 34 & 638.17 & 51.7 & 55.91 & 56.09 & 0.00081 & 1.9 & 336.42 & 131.18 & 0.38 \\
\hline $\begin{array}{l}\text { Samarra } \\
\text { Baghdad }\end{array}$ & 35 & 638.17 & 49.8 & 56.51 & 56.61 & 0.00034 & 1.41 & 453.6 & 145.56 & 0.25 \\
\hline $\begin{array}{l}\text { Samarra } \\
\text { Baghdad }\end{array}$ & 36 & 638.17 & 50.6 & 56.8 & 56.85 & 0.00018 & 1.05 & 609.68 & 184.56 & 0.18 \\
\hline \multirow[t]{2}{*}{$\begin{array}{l}\text { Samarra } \\
\text { Baghdad }\end{array}$} & 37 & 638.17 & 49.4 & 56.88 & 56.93 & 0.00015 & 1 & 639.68 & 182.56 & 0.17 \\
\hline & 38 & $\begin{array}{l}\text { Max } \\
\text { WS }\end{array}$ & 638.17 & 49.4 & 57.01 & 57.02 & 0.000021 & 0.43 & 1475.47 & 342.35 \\
\hline
\end{tabular}

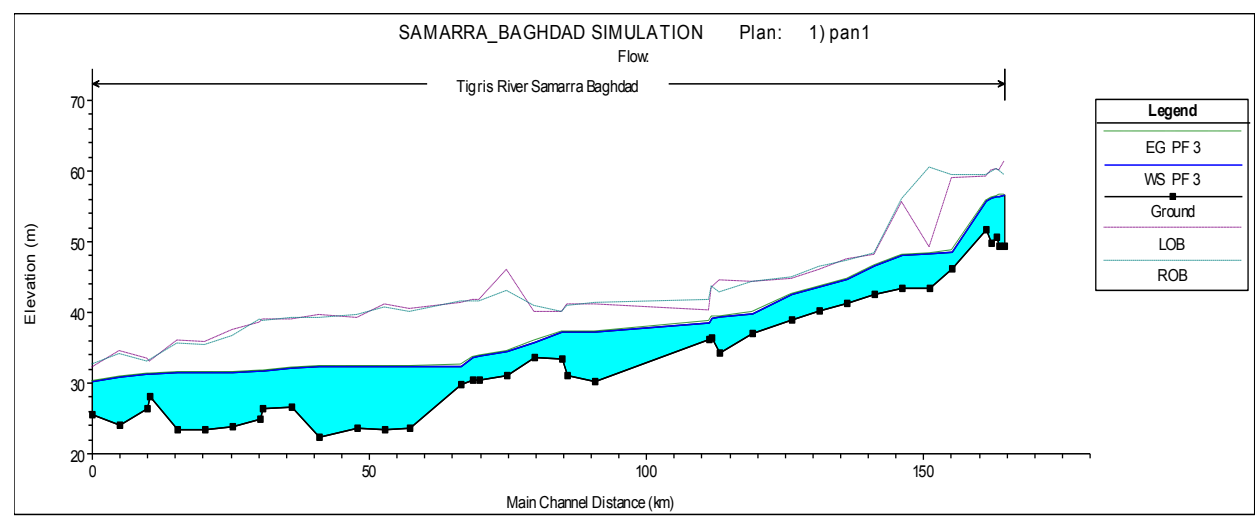

Figure 7: Reach in steady state with Min discharge $(\mathrm{Q}=504.5 \mathrm{~m} / \mathrm{sec})$

Table 6: Output of the Tigris River Unsteady State

\begin{tabular}{|c|c|c|c|c|c|c|c|c|c|c|c|}
\hline Reach & $\begin{array}{c}\text { River } \\
\text { Sta }\end{array}$ & Profile & $\begin{array}{c}\text { Q } \\
\text { Total }\end{array}$ & $\begin{array}{c}\text { Min } \\
\text { Ch } \\
\text { El }\end{array}$ & $\begin{array}{l}\text { W.S. } \\
\text { Elev }\end{array}$ & $\begin{array}{l}\text { E.G. } \\
\text { Elev }\end{array}$ & $\begin{array}{l}\text { E.G. } \\
\text { Slope }\end{array}$ & $\begin{array}{c}\text { Vel } \\
\text { Chnl }\end{array}$ & $\begin{array}{l}\text { Flow } \\
\text { Area }\end{array}$ & $\begin{array}{c}\text { Top } \\
\text { Width }\end{array}$ & $\begin{array}{c}\text { Froude } \\
\text { \# Chl }\end{array}$ \\
\hline & & & $\left(\mathrm{m}^{3} / \mathrm{s}\right)$ & (m) & (m) & (m) & $(\mathrm{m} / \mathrm{m})$ & $(\mathrm{m} / \mathrm{s})$ & $\left(\mathrm{m}^{2}\right)$ & (m) & \\
\hline $\begin{array}{l}\text { Samarra } \\
\text { Baghdad }\end{array}$ & 1 & $\begin{array}{l}\text { Max } \\
\text { WS }\end{array}$ & 635.57 & 25.56 & 30.67 & 30.7 & 0.000142 & 0.78 & 811.75 & 325.22 & 0.16 \\
\hline $\begin{array}{l}\text { Samarra } \\
\text { Baghdad }\end{array}$ & 2 & $\begin{array}{l}\text { Max } \\
\text { WS }\end{array}$ & 635.61 & 24.12 & 31.3 & 31.32 & 0.000106 & 0.65 & 983.38 & 419.06 & 0.13 \\
\hline $\begin{array}{l}\text { Samarra } \\
\text { Baghdad }\end{array}$ & 3 & $\begin{array}{l}\text { Max } \\
\text { WS }\end{array}$ & 635.67 & 26.28 & 31.69 & 31.7 & 0.000052 & 0.43 & 1484.98 & 693.53 & 0.09 \\
\hline $\begin{array}{l}\text { Samarra } \\
\text { Baghdad }\end{array}$ & 4 & $\begin{array}{l}\text { Max } \\
\text { WS }\end{array}$ & 635.68 & 28 & 31.73 & 31.73 & 0.000073 & 0.43 & 1464.13 & 862.95 & 0.11 \\
\hline $\begin{array}{l}\text { Samarra } \\
\text { Baghdad }\end{array}$ & 5 & $\begin{array}{l}\text { Max } \\
\text { WS }\end{array}$ & 635.76 & 23.43 & 31.94 & 31.94 & 0.000011 & 0.23 & 2765.45 & 1013.62 & 0.04 \\
\hline $\begin{array}{l}\text { Samarra } \\
\text { Baghdad }\end{array}$ & 6 & $\begin{array}{l}\text { Max } \\
\text { WS }\end{array}$ & 635.83 & 23.37 & 31.99 & 31.99 & 0.000009 & 0.22 & 2914.1 & 986.59 & 0.04 \\
\hline $\begin{array}{l}\text { Samarra } \\
\text { Baghdad }\end{array}$ & 7 & $\begin{array}{l}\text { Max } \\
\text { WS }\end{array}$ & 635.89 & 23.84 & 32.06 & 32.07 & 0.000021 & 0.38 & 1687.32 & 483.41 & 0.06 \\
\hline $\begin{array}{l}\text { Samarra } \\
\text { Baghdad }\end{array}$ & 8 & $\begin{array}{c}\text { Max } \\
\text { WS }\end{array}$ & 635.92 & 24.79 & 32.15 & 32.16 & 0.000015 & 0.36 & 1787.64 & 433.22 & 0.06 \\
\hline $\begin{array}{l}\text { Samarra } \\
\text { Baghdad }\end{array}$ & 9 & $\begin{array}{l}\text { Max } \\
\text { WS }\end{array}$ & 635.92 & 26.28 & 32.17 & 32.18 & 0.000071 & 0.54 & 1181.75 & 494.33 & 0.11 \\
\hline $\begin{array}{l}\text { Samarra } \\
\text { Baghdad }\end{array}$ & 10 & $\begin{array}{l}\text { Max } \\
\text { WS }\end{array}$ & 635.95 & 26.54 & 32.74 & 32.78 & 0.000168 & 0.88 & 722.3 & 274.79 & 0.17 \\
\hline $\begin{array}{l}\text { Samarra } \\
\text { Baghdad }\end{array}$ & 11 & $\begin{array}{l}\text { Max } \\
\text { WS }\end{array}$ & 635.97 & 22.34 & 33.18 & 33.18 & 0.000001 & 0.12 & 5193.76 & 920.97 & 0.02 \\
\hline
\end{tabular}




\begin{tabular}{|c|c|c|c|c|c|c|c|c|c|c|c|}
\hline $\begin{array}{l}\text { Samarra } \\
\text { Baghdad }\end{array}$ & 12 & $\begin{array}{l}\text { Max } \\
\text { WS }\end{array}$ & 636.02 & 23.54 & 33.19 & 33.19 & 0.000002 & 0.14 & 4489.14 & 967.94 & 0.02 \\
\hline $\begin{array}{l}\text { Samarra } \\
\text { Baghdad }\end{array}$ & 13 & $\begin{array}{l}\text { Max } \\
\text { WS }\end{array}$ & 636.05 & 23.45 & 33.2 & 33.2 & 0.000001 & 0.14 & 4635.67 & 763.66 & 0.02 \\
\hline $\begin{array}{l}\text { Samarra } \\
\text { Baghdad }\end{array}$ & 14 & $\begin{array}{l}\text { Max } \\
\text { WS }\end{array}$ & 636.07 & 23.66 & 33.21 & 33.21 & 0.000006 & 0.34 & 1875.57 & 241.33 & 0.04 \\
\hline $\begin{array}{l}\text { Samarra } \\
\text { Baghdad }\end{array}$ & 15 & $\begin{array}{l}\text { Max } \\
\text { WS }\end{array}$ & 636.71 & 29.85 & 34.32 & 34.38 & 0.000244 & 1.07 & 593.71 & 222.79 & 0.21 \\
\hline $\begin{array}{l}\text { Samarra } \\
\text { Baghdad }\end{array}$ & 16 & $\begin{array}{l}\text { Max } \\
\text { WS }\end{array}$ & 636.72 & 30.46 & 34.71 & 34.74 & 0.000086 & 0.68 & 931.31 & 312.12 & 0.13 \\
\hline $\begin{array}{l}\text { Samarra } \\
\text { Baghdad }\end{array}$ & 17 & $\begin{array}{l}\text { Max } \\
\text { WS }\end{array}$ & 636.73 & 30.46 & 34.8 & 34.82 & 0.000078 & 0.67 & 957.22 & 312.77 & 0.12 \\
\hline $\begin{array}{l}\text { Samarra } \\
\text { Baghdad }\end{array}$ & 18 & $\begin{array}{l}\text { Max } \\
\text { WS }\end{array}$ & 636.78 & 31.14 & 35.2 & 35.22 & 0.00008 & 0.63 & 1016.81 & 368.81 & 0.12 \\
\hline $\begin{array}{l}\text { Samarra } \\
\text { Baghdad }\end{array}$ & 19 & $\begin{array}{l}\text { Max } \\
\text { WS }\end{array}$ & 636.81 & 33.54 & 36.49 & 36.56 & 0.000459 & 1.18 & 541.05 & 282.97 & 0.27 \\
\hline $\begin{array}{l}\text { Samarra } \\
\text { Baghdad }\end{array}$ & 20 & $\begin{array}{l}\text { Max } \\
\text { WS }\end{array}$ & 636.83 & 33.34 & 37.9 & 37.93 & 0.000092 & 0.71 & 897.88 & 299.62 & 0.13 \\
\hline $\begin{array}{l}\text { Samarra } \\
\text { Baghdad }\end{array}$ & 21 & $\begin{array}{l}\text { Max } \\
\text { WS }\end{array}$ & 636.83 & 31.12 & 37.97 & 37.98 & 0.00002 & 0.45 & 1430.76 & 296.64 & 0.06 \\
\hline $\begin{array}{l}\text { Samarra } \\
\text { Baghdad }\end{array}$ & 22 & $\begin{array}{l}\text { Max } \\
\text { WS }\end{array}$ & 636.84 & 30.12 & 38.06 & 38.07 & 0.000014 & 0.32 & 1993.86 & 544.14 & 0.05 \\
\hline $\begin{array}{l}\text { Samarra } \\
\text { Baghdad }\end{array}$ & 23 & $\begin{array}{l}\text { Max } \\
\text { WS }\end{array}$ & 636.89 & 36.26 & 40.3 & 40.34 & 0.000209 & 0.83 & 769.23 & 375.08 & 0.19 \\
\hline $\begin{array}{l}\text { Samarra } \\
\text { Baghdad }\end{array}$ & 24 & $\begin{array}{l}\text { Max } \\
\text { WS }\end{array}$ & 636.89 & 36.36 & 40.4 & 40.44 & 0.000211 & 0.93 & 687.41 & 287.95 & 0.19 \\
\hline $\begin{array}{l}\text { Samarra } \\
\text { Baghdad }\end{array}$ & 25 & $\begin{array}{l}\text { Max } \\
\text { WS }\end{array}$ & 637 & 34.23 & 40.6 & 40.6 & 0.000017 & 0.35 & 1806.03 & 493.31 & 0.06 \\
\hline $\begin{array}{l}\text { Samarra } \\
\text { Baghdad }\end{array}$ & 26 & $\begin{array}{l}\text { Max } \\
\text { WS }\end{array}$ & 637.13 & 37.11 & 41.09 & 41.13 & 0.000157 & 0.8 & 793.45 & 329.95 & 0.17 \\
\hline $\begin{array}{l}\text { Samarra } \\
\text { Baghdad }\end{array}$ & 27 & $\begin{array}{l}\text { Max } \\
\text { WS }\end{array}$ & 637.28 & 39 & 42.78 & 42.82 & 0.000336 & 0.98 & 652.08 & 356.9 & 0.23 \\
\hline $\begin{array}{l}\text { Samarra } \\
\text { Baghdad }\end{array}$ & 28 & $\begin{array}{l}\text { Max } \\
\text { WS }\end{array}$ & 637.47 & 40.22 & 44.09 & 44.11 & 0.000212 & 0.61 & 1041.29 & 815.43 & 0.17 \\
\hline $\begin{array}{l}\text { Samarra } \\
\text { Baghdad }\end{array}$ & 29 & $\begin{array}{c}\text { Max } \\
\text { WS }\end{array}$ & 637.67 & 41.37 & 45.11 & 45.14 & 0.000223 & 0.77 & 825.94 & 473.78 & 0.19 \\
\hline $\begin{array}{l}\text { Samarra } \\
\text { Baghdad }\end{array}$ & 30 & $\begin{array}{l}\text { Max } \\
\text { WS }\end{array}$ & 637.8 & 42.45 & 46.85 & 46.88 & 0.000479 & 0.81 & 791.48 & 755.97 & 0.25 \\
\hline $\begin{array}{l}\text { Samarra } \\
\text { Baghdad }\end{array}$ & 31 & $\begin{array}{l}\text { Max } \\
\text { WS }\end{array}$ & 637.89 & 43.3 & 48.44 & 48.47 & 0.00016 & 0.77 & 833.31 & 374.61 & 0.16 \\
\hline $\begin{array}{l}\text { Samarra } \\
\text { Baghdad }\end{array}$ & 32 & $\begin{array}{l}\text { Max } \\
\text { WS }\end{array}$ & 637.98 & 43.3 & 48.9 & 48.9 & 0.000016 & 0.29 & 2172.78 & 744.11 & 0.05 \\
\hline $\begin{array}{l}\text { Samarra } \\
\text { Baghdad }\end{array}$ & 33 & $\begin{array}{c}\text { Max } \\
\text { WS }\end{array}$ & 638.06 & 46.1 & 49.62 & 49.68 & 0.000364 & 1.05 & 606.13 & 315.45 & 0.24 \\
\hline $\begin{array}{l}\text { Samarra } \\
\text { Baghdad }\end{array}$ & 34 & $\begin{array}{l}\text { Max } \\
\text { WS }\end{array}$ & 638.12 & 51.7 & 55.34 & 55.64 & 0.001621 & 2.42 & 264.01 & 120.68 & 0.52 \\
\hline $\begin{array}{l}\text { Samarra } \\
\text { Baghdad }\end{array}$ & 35 & $\begin{array}{l}\text { Max } \\
\text { WS }\end{array}$ & 638.13 & 49.8 & 56.5 & 56.6 & 0.000346 & 1.41 & 452.71 & 145.5 & 0.26 \\
\hline $\begin{array}{l}\text { Samarra } \\
\text { Baghdad }\end{array}$ & 36 & $\begin{array}{l}\text { Max } \\
\text { WS }\end{array}$ & 638.14 & 50.6 & 56.81 & 56.86 & 0.000175 & 1.04 & 611.43 & 184.82 & 0.18 \\
\hline $\begin{array}{l}\text { Samarra } \\
\text { Baghdad }\end{array}$ & 37 & $\begin{array}{l}\text { Max } \\
\text { WS }\end{array}$ & 638.15 & 49.4 & 56.89 & 56.94 & 0.000147 & 1 & 641.34 & 182.78 & 0.17 \\
\hline $\begin{array}{l}\text { Samarra } \\
\text { Baghdad }\end{array}$ & 38 & $\begin{array}{l}\text { Max } \\
\text { WS }\end{array}$ & 638.17 & 49.4 & 57.01 & 57.02 & 0.000021 & 0.43 & 1475.47 & 342.35 & 0.07 \\
\hline
\end{tabular}

\section{Acknowledgment}

The authors would wish to thank the technical and faculty staff of the Department of Civil Engineering at the University of Technology-Iraq for the valuable support and scientific assistance. Also, we would like to express our appreciation to the Ministry of Water Resources/National Centre of Water Resources Management, Iraq for their in facilitating our research task through providing the required data and conducting the field study.

\section{Author contribution}

All authors contributed equally to this work

\section{Funding}

This research received no specific grant from any funding agency in the public, commercial, or not-for-profit sectors.

Data availability statement

The data that support the findings of this study are available on request from the corresponding author.

\section{Conflicts of interest}

The authors declare that there is no conflict of interest. 


\section{References}

[1] Arun, D. I., Aryadutt, O., \& Prajith, K. K. P. Aerodynamic Decelerator Atmospheric-Entry Module (ADAM) FOR MARS. ISHMT Digital Library. Begel House Inc,2017.

[2] Allison, J., Amako, K., Apostolakis, J., Arce, P., Asai, M., Aso, T. Barrand, G. Recent developments in Geant4. Nuclear Instruments and Methods in Physics Research Section A: Accelerators, Spectrometers, Detectors and Associated Equipment, 835(2016)186-225. https://doi.org/10.1016/j.nima.2016.06.125

[3] Mahmoud Saleh Al-Khafaji . Evaluating the Hydraulic Performance of Al Msharah River, Eng. Tech., $26(2008) 338$.

[4] Ala Hassan Nama . Estimating the Sediment Transport Capacity of Tigris River Within Al Mosul City. J. Eng., 17(2011)473.

[5] Ala Hassan Nama and Zahraa Abdulhussain, Riverbed Scour Due to Accumulation of Floating Debris onAl-Msharah Bridge Piers. Al-Nahrain University, College of Engineering Journal (NUCEJ) 18 ( 2015) 16 -25.

[6] Ala Hassan Nama. Distribution of Shear Stress in the Meanders of Tigris River Within Baghdad City. Al-Nahrain University, College of Engineering Jou rnal (NUCEJ).18 (2015)26 - 40.

[7] Abdelbasset, Midaoui and Lahrach A. and Chaouni_Adel A. and Bourak A. and Benaabidate L., Integration of GIS and HE-RAS in flood modeling of the ouergha river, northem Morocco, 11(2015).

[8] Dang \& K.Lalit, Application 0f remote sensing and GIS-based hydrological modelling for flood risk analysis:a case study of District8, Ho Chi Minh city, Vietnam, Published by Informa UK Limited, trading as Taylor Francis Groop,2017.,

[9] Sharma and Mujumdar Floodplain Zoning Simulation by Using HEC-RAS and CCHE2D Models in the Sungai Maka River'

[10] Traore \&others, Using of HEC-RAS Model for Hydraulic Analysis of a river with Agriculture Vocation :A CaseStudy of the Kayanga River Basin, Senegal, JAWRA., 3 (2015)147-154. https://doi.org/10.12691/ajwr-3-5-2

[11] F. Onen and T. Bagatur, Prediction of flood frequency factor for Gumbel distribution using regression and GEP model, AJSE., 42(2017) 3895-3906.

[12] T. Bagatur and F. Onen, Development of predictive model for flood routing using genetic expression programming, J. Flood. Risk. Manag ., 11( 2018) 444-454. https://doi.org/10.1111/jfr3.12232

[13] N. S. Romali, Z. Yusop, and A. Z. Ismail, Application of HEC-RAS and Arc GIS for floodplain mapping in Segamat town, Malaysia, Int. J. GEOMATE., 14(2018)125-131. https://doi.org/10.21660/2018.43.3656

[14] A. Imzahim A, Z. W Samueel, and Q. K Abdullah. Accuracy Assessment of Digital Elevation Models Produced From Different Geomatics Data, Eng. Technol. J., 38 ( 2020)1580-92. DOI: https://doi.org/10.30684/etj.v38i11A.1318

[15] Maatooq, Jaafar S, and Luay K Hameed. 2D Model to Investigate the Morphological and Hydraulic Changes of Meanders, Eng. Technol. J ., 38 (2020)9-19. https://doi.org/10.30684/etj.v38i1A.95

[16] Karim, Ibtisam R, and Sarmad A Sahib. The Hydraulic Analysis of The Irrigation Network of The Proposed Taq-Taq Dam Using EPANET Software, Eng. Technol. J., 37 (2019) 429-34. https://doi.org/10.30684/etj.37.10A.8

[17] C. B. Khalfallah and S. Saidi, Spatiotemporal floodplai mapping and prediction using HEC-RAS-GIS tools: case of the Mejerda river, Tunisia, J. Afr. Earth. Sci., 142(2018)44-51. https://doi.org/10.1016/j.jafrearsci.2018.03.004

[18] Hossein, A., Javaheri, N., Daghigh, Y., \& Tolooiyan, A. Simulating of Unsteady Flow in Tidal Zones By Using HECRAS Model, Strömungssimulation Im Wasserbau Flow Simulation in Hydraulic Engineering, 32 (2006)57-65.

[19] Rabies, A. M., Abdul-Kareem, B. M., \& Al-Dhamin, A. S. Seasonal variations of some ecological parameters in Tigris River water at Baghdad Region, Iraq, JWARP., 3(2011)262. https://doi.org/10.4236/jwarp.2011.34033

[20] G. W. Brunner, HEC-RAS River Analysis System Hydraulic Reference Manual, Version 4.1, U.S. Army Corps of Engineers, Washington, DC, USA, 2010.

[21] The Ministry of Water Resources.

[22] Hec-Ras, Hydraulic Engineering Center, River Analysis System, User Manual2002. 